Gefässchirurgie 2022 $27: 39-45$

https://doi.org/10.1007/s00772-021-00855-0

Angenommen: 3. Dezember 2021

Online publiziert: 23. Dezember 2021

(c) Der/die Autor(en) 2021
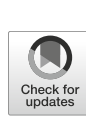

\section{Empfehlungen zur konservativen Therapie und Sekundär- prävention der peripheren arteriellen Verschlusskrankheit (PAVK): Eine evidenzbasierte Informationsbroschüre für Betroffene}

\author{
Yvonne Rosenberg $\cdot$ Hartmut Görtz ${ }^{2,7}$. Ulrich Rother ${ }^{3,7}$. Christian Uhl ${ }^{4,7}$. \\ Konstantinos Stavroulakis ${ }^{5,7} \cdot$ Manfred Pfeiffer $^{6} \cdot$ Mechtild Pfeiffer-Krahl $^{6}$. \\ Christian-Alexander Behrendt ${ }^{1,7}$ \\ 'Forschungsgruppe GermanVasc, Klinik und Poliklinik für Gefäßmedizin, Universitätsklinikum Hamburg- \\ Eppendorf, Hamburg, Deutschland; ${ }^{2}$ Klinik für Gefäßchirurgie, Bonifatius Hospital Lingen, Lingen, \\ Deutschland; ${ }^{3}$ Gefäßchirurgische Abteilung, Universitätsklinikum Erlangen, Erlangen, Deutschland; \\ ${ }^{4}$ Klinik für Gefäßchirurgie und Endovaskuläre Chirurgie, Universitätsklinikum Heidelberg, Heidelberg, \\ Deutschland; ${ }^{5}$ Klinik für Gefäßchirurgie, Ludwig-Maximilians-Universitätsklinikum, München, \\ Deutschland; ${ }^{6}$ Sektion AVK-Selbsthilfe-\&-Rehasport, Das PatientenForum e.V., Sörgenloch, Deutschland; \\ ${ }^{7}$ Kommission PAVK und Diabetisches Fußsyndrom der Deutschen Gesellschaft für Gefäßchirurgie und \\ Gefäßmedizin e.V., Berlin, Deutschland
}

\title{
Zusammenfassung
}

Die periphere arterielle Verschlusskrankheit (PAVK) gilt als Volkskrankheit mit weltweit mehr als 230 Mio. Betroffenen und schlechter Prognose. Durch den systemischen und progressiven Charakter der Atherosklerose sowie den Befall vieler Gefäßbereiche ist neben dem Risiko für gefäßbedingte Amputationen auch die generelle Lebenserwartung deutlich eingeschränkt. Die strikte Ausschöpfung der konservativen Therapie gilt als wichtiges Fundament der komplementären Behandlung, wird aber nicht immer erfolgreich umgesetzt. Neben dem strukturierten Gehtraining, Raucherentwöhnung, Optimierung von Ernährung und Körpergewicht, Blutdrucktherapie sowie Normalisierung von Blutzucker- und Blutfettwerten gilt die optimale Arzneimitteltherapie als zentrales Behandlungsziel. Dieser Artikel soll laienverständliche evidenzbasierte Empfehlungen zur Optimierung des sogenannten Best Medical Treatment in der Behandlung der PAVK geben.

\section{Schlüsselwörter}

Periphere arterielle Verschlusskrankheit · Prävention · Atherosklerose - Claudicatio intermittens . Arzneimitteltherapie

\section{Einleitung}

Die periphere arterielle Verschlusskrankheit (PAVK), im Volksmund oft "Schaufensterkrankheit" genannt, beruht auf einer krankhaften Einengung von Blutgefäßen (Schlagadern). Die Erkrankung entsteht meist auf dem Boden einer Atherosklerose (Gefäßverkalkung) und kann grundsätzlich alle Gefäße im Körper betreffen. Wenn die Beinarterien betroffen sind, führt dies in fortgeschrittenen Stadien zu Muskelschmerzen beim Gehen (z. B. in den Waden) oder sogar zu durch Sauerstoffmangel bedingte Ruheschmerzen ohne Belastung oder Gewebeuntergang mit/ohne Infektion (Nekrose/Gangrän) (- Abb. 1). Daneben sind nicht selten auch 


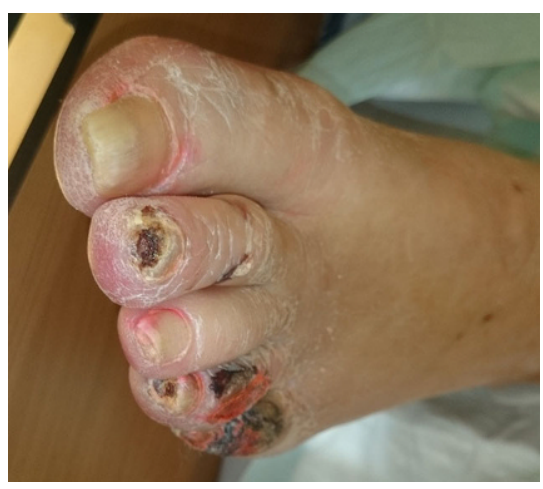

Abb. 1 A Trockene Zehennekrosen des 2., 4. und 5. Zehs am linken Fuß bei Vorliegen einer chronischen extremitätengefährdenden Ischämie (Fontaine-Stadium IV)

die Gefäße zur Versorgung des Herzens, Gehirns und der inneren Organe betroffen. Die PAVK unterscheidet sich dabei nicht wesentlich von der Atherosklerose der Herzkranzgefäße und oft sind beide Organbereiche betroffen[19]. Durch diesen Befall mehrerer Gefäßbereiche haben Patientinnen und Patienten mit PAVK ein vielfach erhöhtes Risiko, an einem Herzinfarkt oder Schlaganfall zu versterben $[32,47]$. Insgesamt betrifft das Fünfjahresrisiko zu versterben oder eine Amputation zu erleiden, je nach Krankheitsstadium und Risikoprofil zwischen 9 und $88 \%$ (- Abb. 2, https://score.germanvasc.de). Weltweit sind von dieser Krankheit mehr als 230 Mio. Menschen betroffen und die PAVK gilt auch in Deutschland mittlerweile als Volkskrankheit [22, 32, 33, 49].

\section{॥ Die PAVK ist eine häufige Volkskrankheit mit weltweit mehr als 230 Mio. Betroffenen}

Es gibt dabei Risikofaktoren für eine PAVK, auf die man keinen Einfluss hat, wie z.B. das Alter, genetische Veranlagung und Geschlecht. Andere Risikofaktoren sind hingegen beeinflussbar. Dazu zählen: Das Rauchen, Bewegungsmangel, ungesunde Ernährung und Übergewicht, Bluthochdruck, Störungen des Fettstoffwechsels (Dyslipidämie) sowie des Zuckerstoffwechsels (Diabetes) [1, 21, 25].

Die Therapie der PAVK beruht grundsätzlich auf drei Säulen, die je nach Krankheitsstadium einzeln oder kombiniert eingesetzt werden: Ab der Erstdiagnose einer PAVK sollte die konservative Thera-

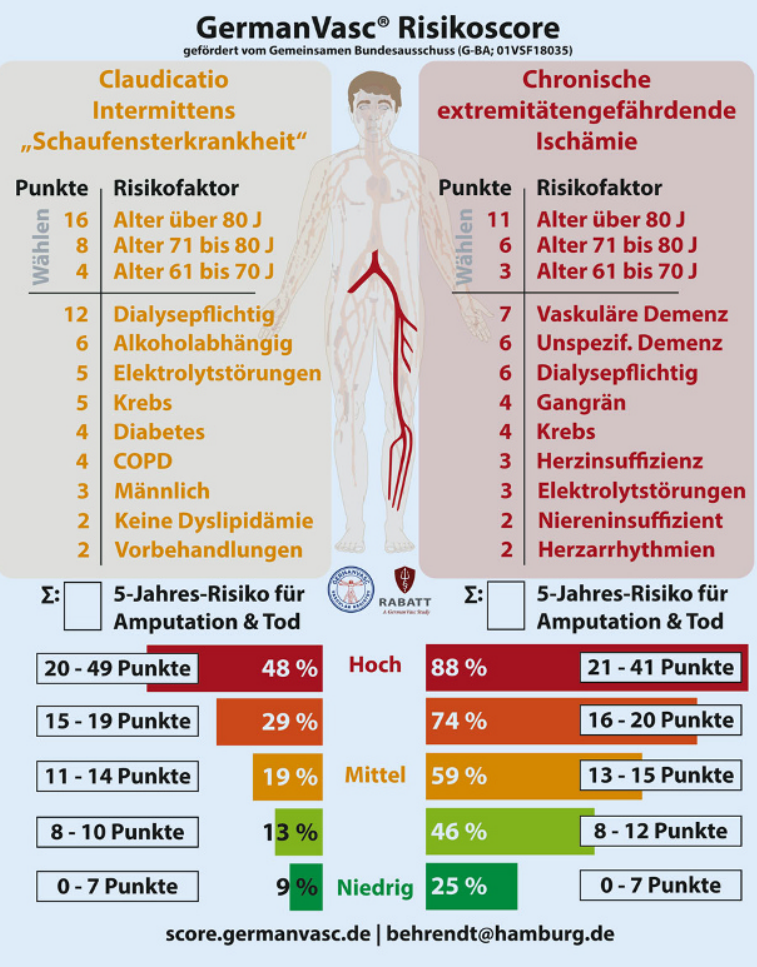

Abb. $2 \triangleleft$ GermanVasc-Risikoscore zur Vorhersage des Fünfjahresrisikos für das Auftreten von Amputation oder Tod. Quelle: https:// score.germanvasc. de. Je nach Krankheitsstadium (links und rechts) erzeugen die verschiedenen Risikofaktoren jeweils einen Punktwert. Die Gesamtsumme entspricht einer der fünf Risikogruppen (unten), adaptiert nach [32]

pie konsequent durchgeführt werden, z. B. das strukturierte Gehtraining, das in fortgeschrittenen symptomatischen Stadien durch invasive durchblutungsverbessernde Verfahren ergänzt werden kann. Mit dem Begriff konservative Therapie beschreibt man heutzutage die Behandlung der beeinflussbaren Risikofaktoren [10] durch Verabreichung von Arzneimitteln und andere Maßnahmen, die sich bei einer PAVK als effektiv erwiesen haben [39]. Die Optimierung der Risikofaktoren kann dabei nicht nur die Entstehung, sondern auch die Weiterentwicklung bzw. die Prognose der PAVK [34] verbessern und verlängert insgesamt das Überleben [47].

Im weiteren Inhalt dieser Broschüre werden die einzelnen Risikofaktoren genauer beleuchtet und Empfehlungen zur Behandlung gegeben.

\section{Rauchen}

Rauchen ist der bedeutendste Risikofaktor für die Entstehung und den Verlauf der PAVK. Dabei hat die Menge der gerauchten Zigaretten und die Dauer des Konsums einen negativen Einfluss auf die
Schwere der Erkrankung und auch auf die Sterblichkeit [34].

I) Rauchen ist der bedeutendste Risikofaktor für die Entstehung und den Verlauf der PAVK

Zigarettenrauch schädigt die Blutgefäße und führt so zur Entwicklung der Atherosklerose [43].

Mit dem Rauchen aufzuhören, senkt deshalb auch die Wahrscheinlichkeit, neben der PAVK eine koronare Herzkrankheit (KHK) zu entwickeln, zu erkranken oder einen Schlaganfall zu erleiden [17]. Das Risiko, an einer Demenz (z. B. Alzheimer) zu erkranken, ist bei Rauchern um 30 bis $40 \%$ erhöht. Auch die Wundheilung und die Funktion des Immunsystems werden durch den Tabakkonsum beeinträchtigt („Raucherbein“). Innerhalb von 10 Jahren nach der Erstdiagnose einer PAVK ist bei 4 bis $7 \%$ der Frauen bzw. Männer Lungenkrebs diagnostiziert worden, der auf die hohe Rate des Tabakkonsums zurückgeführt wird [30].

Daher raten wissenschaftliche Fachgesellschaften übereinstimmend dazu, mit dem Rauchen aufzuhören und beziehen 
sich dabei auf hochwertige wissenschaftliche Arbeiten [8, 24, 26, 34].

Sie können mit Ihrem Arzt oder Ihrer Ärztin über Hilfsmittel zur Rauchentwöhnung sprechen, beispielsweise können überbrückend auch Medikamente in Verbindung mit Entwöhnungsprogrammen besonders hilfreich sein [26]. Weitere Infomaterialien und Unterstützung können Sie z.B. bei der Bundeszentrale für gesundheitliche Aufklärung (BZgA) finden (https://www.bzga.de/).

\section{Ernährung und Körpergewicht}

Betroffenen einer PAVK wird eine gesunde und ausgewogene Ernährung empfohlen $[2,13,24]$.

Ein Beispiel für eine gesunde Ernährung ist die mediterrane Ernährung (mediterrane Diät), welche durch einen hohen Anteil an pflanzlichen Nahrungsmitteln gekennzeichnet ist. Dazu gehören der tägliche Konsum von Olivenöl, Nüssen, Früchten und Gemüse, Leinsamen, aber auch von Produkten aus Vollkorngetreide. Gleichzeitig ist der Verzehr von Milchprodukten, Eiern und auch Geflügel und Meeresfrüchten moderat (d. h. nur wenige Tage pro Woche). Rotes und verarbeitetes Fleisch und Fisch sowie süße Nachtische und gesüßte Getränke (Wasser ist das Getränk der Wahl) werden nur im geringen Maße konsumiert [46]. Studien haben gezeigt, dass die mediterrane Ernährung zu einer Reduktion von kardiovaskulären Risiken und zu einer besseren Prognose führt $[3,12,14,37,46]$.

\section{॥) Die mediterrane Diät kann die Entwicklung einer PAVK verlangsamen und den Verlauf positiv beeinflussen}

In der mediterranen Ernährung enthaltene, mehrfach ungesättigte Fettsäuren haben beispielsweise einen positiven Einfluss auf die Prävention der PAVK [31, 35]. Diese können in Form von pflanzlichen Ölen und Nüssen den Mahlzeiten beigefügt werden.

Auch Ballaststoffe, z. B. viel in Gemüse und anderen pflanzlichen Produkten vorhanden, haben positive Effekte auf den Blutzuckerhaushalt und reduzieren Blutfette und chronische Entzündungen [6]. Leinsamen wirkt sich ebenfalls po- sitiv auf kardiovaskuläre Risikofaktoren, Blutfette und den Blutdruck aus [11, 20, 45]. Zusätzlich wird eine Reduktion des Salzkonsums auf maximal $5 \mathrm{~g}$ Salz pro Tag empfohlen, da eine höhere Aufnahme mit einem Bluthochdruck in Verbindung steht. Wichtig ist zu wissen, dass sich $80 \%$ des täglich aufgenommenen Salzes in bereits verarbeiteten Lebensmitteln befinden und daher in die Berechnung einbezogen werden müssen.

Ergänzend wird bei Übergewicht eine Gewichtsreduktion empfohlen [34]. Zu den Folgen von Übergewicht zählen ein Anstieg des Blutdrucks, eine Resistenz gegenüber Insulin und dadurch die Entwicklung eines Typ-2-Diabetes (metabolisches Syndrom), erhöhte Blutfette, chronische Entzündungen im Körper und zusätzlich die Entwicklung von Thrombosen (Blutgerinnseln) [42].

\section{I) Übergewicht und Fettleibigkeit sind mit Bluthochdruck und Störungen des Zuckerstoffwechsels assoziiert}

Sowohl Übergewicht als auch Fettleibigkeit erhöhen das Risiko, an einem kardiovaskulären Ereignis zu versterben [52]. Eine Gewichtsreduktion verbessert die Blutfette [54], den Blutdruck [52] und den Langzeitblutzucker (HbA1c) [16].

Ziel ist gemäß Weltgesundheitsorganisation (WHO) ein Body-Mass-Index (BMI) von $20 \mathrm{~kg} / \mathrm{m}^{2}$ bis $25 \mathrm{~kg} / \mathrm{m}^{2}$ bei unter 60 Jährigen (im Alter dürfen die Werte etwas höher sein, z.B. ein Körpergewicht von max. $80 \mathrm{~kg}$ bei $1,75 \mathrm{~m}$ Körpergröße) und ein Taillenumfang von weniger als $94 \mathrm{~cm}$ für Männer bzw. weniger als $80 \mathrm{~cm}$ fürFrauen [42]. Zur Verbesserung der Ernährung wird eine begleitende und langfristige Ernährungsberatung empfohlen.

\section{Gehtraining}

Basistherapie nach den S3-Leitlinien der Arbeitsgemeinschaft der Wissenschaftlichen Medizinischen Fachgesellschaften (AWMF) zur Diagnostik, Therapie und Nachsorge der PAVK ist generell das strukturierte Gehtraining, insbesondere bei Vorliegen durchblutungsbedingter belastungsabhängiger Muskelschmerzen (Claudicatio intermittens). Auch nach einer Gefäßrekonstruktion wird zur weiteren Verbesserung der Durchblutung über Umgehungskreisläufe (Kollateralen) in der Regel ein strukturiertes Gehtraining empfohlen [24]. Im Vergleich zu einer der beiden Optionen allein zeigt die Kombination aus einer operativen Therapie und Gehtraining bessere Ergebnisse auf die schmerzfreie Gehstrecke und die krankheitsbezogene Lebensqualität [26, 29, 38, 48]. Zusätzlich wird die Ausdauer gesteigert und die kardiovaskuläre Sterblichkeit sinkt durch regelmäßige körperliche Aktivität [34]. Es gibt Hinweise darauf, dass ein Gehtraining die Entzündung im Körper beeinflusst und positiv auf die Gefäßfunktion sowie Kapillardurchblutung wirken kann. Durch eine Zunahme wichtiger Enzyme kann auch die Zusammensetzung der Muskulatur verändert werden [28, 44, 50].

Erfolgreich kann ein eigenständiges Training sein, noch besser eignet sich ein überwachtes Training, das durch von den Landessportverbänden ausgebildete Übungsleiter und Übungsleiterinnen angeboten wird.

Das PatientenForum e. V., Sektion AVKSelbsthilfe-\&-Rehasport tritt hier als Berater auf und kann jederzeit angesprochen werden (info@das-patientenforum.de).

Von wesentlicher Bedeutung ist, dass das Training regelmäßig erfolgt. Für den Umfang eines selbstständigen Trainings werden ca. 60 Minuten täglich empfohlen. Innerhalb dieses Trainings sollte es zu 5- bis 15-minütigen Belastungsspitzen kommen, bis Schmerzen auftreten [34]. Im Anschluss sollte kurz geruht werden, bis der Schmerz vollständig verschwunden ist und erst im Anschluss sollte weiter trainiert werden. Nordic Walking ist zu empfehlen, da es durch die Stöcke die Belastung auf die Beine senkt und gut für den Stoffwechsel ist [34]. Falls beim Training die Schmerzen subjektiv nicht erträglich sind, kann auch ein schmerzfreies Training versucht werden [26]. Auch andere Formen des Trainings können genutzt werden [26].

Gehtraining wird als Rehabilitationsmaßnahme von einem Arzt oder einer Ärztin verordnet und wird von den Krankenkassen bezahlt. 


\section{Arzneimitteltherapie}

Die optimale Arzneimitteltherapie bei PAVK besteht grundsätzlich aus drei Substanzklassen:

- den Blutdrucksenkern,

- den Blutfett-bzw. Lipidsenkern und

- den Thrombozytenaggregationshemmern ("Gerinnungshemmer").

Diese Arzneimittelgruppen verringern die Rate an Amputationen und verlängern das Überleben, indem sie dazu beitragen kardiovaskuläre Ereignisse (z. B. Herzinfarkte oder Schlaganfälle) zu verhindern [1, 25, $39,40]$.

\section{Arzneimittel zur Bluthochdruck- therapie}

Begleitend zur PAVK liegt oft auch eine arterielle Hypertonie (Bluthochdruck) vor. Internationale Leitlinien empfehlen, den Bluthochdruck konsequent zu behandeln $[2,26,34]$. Dabei soll der Blutdruck dauerhaft auf $<140 / 90 \mathrm{~mm} \mathrm{Hg}$ gesenkt werden [51]. Ein vorsichtiges Vorgehen ist dabei sinnvoll, da eine rasche Absenkung des bestehenden Blutdrucks insbesondere initial auch zu unerwünschten Ereignissen (z. B. Schwindel, Sturz) führen kann [2]. Es gibt verschiedene Wirkstoffklassen zur Therapie. Die Entscheidung über den spezifischen Wirkstoff hängt von individuellen Faktoren wie Vorerkrankungen, Unverträglichkeiten und auch dem Alter ab. In der Regel wird die Therapie mit sogenannten ACE-Hemmern (Angiotensinkonversionsenzymhemmer z.B. Ramipril) [2, 34] bzw. Angiotensinrezeptorblockern (z.B. Candesartan) [24] und Calciumkanalblockern [34] als Kombinationspräparat begonnen [51]. Leichte Unverträglichkeiten können in der Regel durch den Wechsel der Präparate behoben werden $[7,41]$. Letztendlich muss die Wahl des richtigen Blutdrucksenkers bzw. von dessen Kombination in Zusammenarbeit mit dem zuständigen Haus- oder Facharzt bzw. der Fachärztin erfolgen.

\section{Arzneimittel zur Blutfettsenkung}

Eine wichtige Arzneimittelgruppe stellen die Blutfettsenker dar. Leitlinien empfehlen einvernehmlich, dass jede Patientin und jeder Patient mit einer PAVK, unabhängig vom Vorliegen einer Symptomatik, einen Blutfettsenker erhalten soll [1, 13, 25]. Die risikoadaptierten Zielwerte für das sogenannte LDL-Cholesterin als Marker für die Fettstoffwechselstörung sind in den letzten Jahren auf dem Boden neuerer Arzneimittelstudien immer weiter gesenkt worden $(<55 \mathrm{mg} / \mathrm{dl}$ für Patienten mit PAVK) [36]. Dabei sind die sogenannten Statine, ggf. ergänzt durch das Präparat Ezetimib, weiterhin das Mittel der ersten Wahl $[2,26,34]$. Sie hemmen in der Leber ein Schlüsselenzym und damit die Herstellung von Cholesterin [36]. Grundsätzlich empfiehlt es sich, die Tabletten abends einzunehmen, unter anderem weil nachts am meisten Cholesterin vom Körper produziert wird [36].

Statine senken die Wahrscheinlichkeit, ein kardiovaskuläres Ereignis zu erleiden oder daran zu versterben im Langzeitverlauf um bis zu $30 \%[34,36]$.

\section{I) Statine sind ein wesentlicher Baustein in der optimalen Arzneimitteltherapie der PAVK}

Insgesamt sind Statine sehr gut verträgliche und sichere Medikamente [36] schwere unerwünschte Nebenwirkungen sind sehr selten und lassen sich häufig durch einen Präparatewechsel beheben. Meistens sind auftretende Beschwerden nicht auf die Einnahme des Statins zurückzuführen [27, 53]. Wichtig ist es, über jegliche auftretenden Probleme mit dem zuständigen Arzt oder der Ärztin zu sprechen und eine gemeinsame Lösung zu finden. In der Regel kann der Wechsel auf ein besser verträgliches Statin bereits helfen.

\section{Arzneimittel zur Hemmung der Blutplättchen}

Bei einer symptomatischen PAVK gibt es eine klare Empfehlung zur Einnahme von Clopidogrel (75 mg) oder Aspirin (100 mg) zur Reduktion von kardiovaskulären Ereignissen [2, 26, 34]. Bei Vorliegen anderer Erkrankungen, z.B. von Vorhofflimmern oder nach erfolgten Gefäßrekonstruktionen kann auch deren Kombination („doppelte Plättchenhemmung ") oder eine intensivierte gerinnungswirksame Medi- kation bzw. Blutverdünnung empfohlen werden. Insgesamt sorgt eine Hemmung der Blutgerinnung dafür, dass die Wahrscheinlichkeit für weitere Eingriffe sinkt [9]. Bei der Empfehlung zur gerinnungswirksamen Medikation wird der erwartete Nutzen immer dem möglichen Blutungsrisiko gegenübergestellt [23]. Meistens handelt es sich hierbei um Blutungen des oberen Magen-Darm-Traktes. Vorbeugend wird daher meist auch ein Medikament zum Schutz der Magenschleimhaut (PPI, Protonenpumpenhemmer) verordnet [23].

Ein Plättchenhemmer, etwa Clopidogrel oder Aspirin in niedriger Dosierung, oder eine Kombination aus Aspirin und Rivaroxaban stellen heutzutage eine wichtige Säule der Arzneimitteltherapie der PAVK dar.

\section{Diabetes}

Wenn man an einem Diabetes erkrankt ist, ist das Risiko auch an einer PAVK zu erkranken, um das Drei- bis Vierfache erhöht [24]. Zusätzlich verschlechtert ein Diabetes bei einer PAVK die Gehleistung [18], sorgt für ein Fortschreiten der PAVK, erhöht das Risiko für Amputationen und die Sterblichkeit [26].

Wer bereits an einer PAVK erkrankt ist, profitiert trotzdem von stabileren Blutzuckerwerten, um das Voranschreiten der PAVK zu reduzieren [24]. Diabetes ist hinter dem Rauchen der zweitwichtigste Risikofaktor für die Verschlechterung der PAVK [34].

Ein niedrigerer $\mathrm{HbA1c}$-Wert als Marker für den Langzeitblutzucker unter $7 \%$ führt außerdem zu einer geringeren Amputationsrate [4].

Die Zielwerte für den $\mathrm{HbA} 1 \mathrm{c}$ sind $\mathrm{ab}$ hängig vom Alter, Begleiterkrankungen und dem Hypoglykämierisiko (Risiko zur Unterzuckerung). Der ideale Zielkorridor liegt zwischen 6,5 und $7,5 \%$, im höheren Alter zwischen 7 und $8 \%[5,15,34]$. Genaue und individualisierte Zielwerte sollten mit behandelnden Ärztinnen und Ärzten besprochen werden.

Eine Senkung des Blutzuckers kann durch Medikamente in Verbindung mit einer Veränderung des Lebensstils erreicht werden. Meist ist eine Kombination aus einer Diät und Arzneimitteltherapien sinnvoll, wobei in der Regel eine stu- 


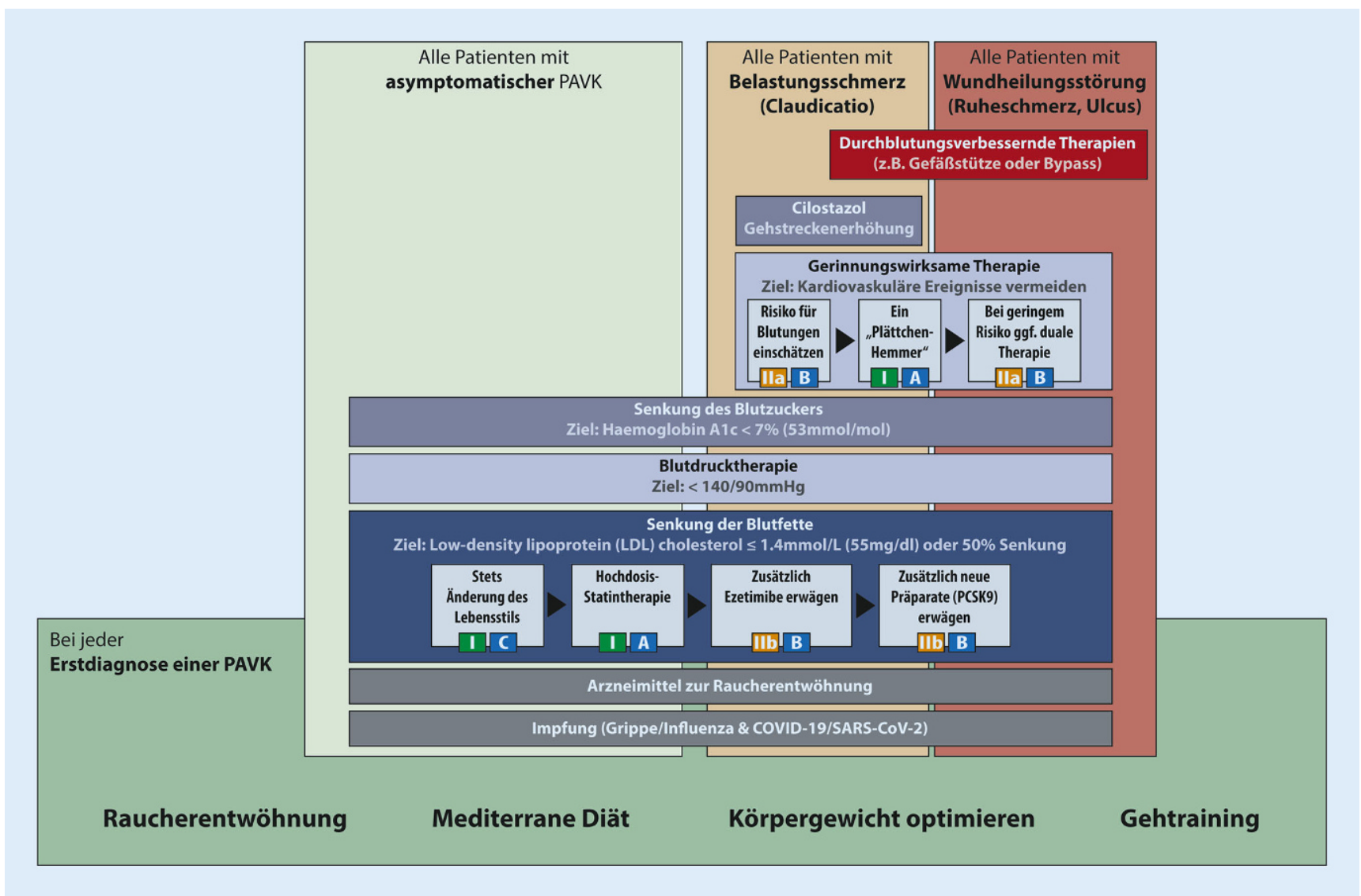

Abb. 3 ॥ Stufenbasierte Therapie der peripheren arteriellen Verschlusskrankheit (PAVK)

fenweise Steigerung der Bemühungen verfolgt wird.

\section{॥) Diabetes ist nach dem Rauchen der zweitwichtigste Risikofaktor für die Verschlechterung einer PAVK}

Bei einem Diabetes kann zusätzlich eine Polyneuropathie (nervenbedingter Gefühlsverlust) z. B. in den Beinen und Füßen vorliegen. Diese führt unter Umständen dazu, dass kleine Wunden, Schmerzen oder eine verschlechterte Durchblutung der betroffenen Gliedmaßen nicht bemerkt werden [34].

Ein weiterer Teil der Diabetesbehandlung müssen deshalb regelmäßige medizinische Fußpflege und eine Prophylaxe tiefer Wunden (Ulcusprophylaxe) sein [26]. Wichtig ist, dass Patientinnen und Patienten mit einem Diabetes auf die Vermeidung von Druckstellen durch falsches Schuhwerk achten. Außerdem sollte nur eine medizinische Fußpflege in Anspruch genommen werden.

\section{Diskussion}

Der Verlauf Ihrer Erkrankung und damit auch die Lebensqualität lassen sich durch eine konsequente und umfassende konservative Therapie spürbar verbessern. Es ist wichtig, dass Sie den Inhalt und die Bedeutung dieser Empfehlungen verstanden haben; und es ist die Verantwortung der behandelnden Ärztinnen und Ärzte, Ihnen diese Informationen in verständlicher Weise zur Verfügung zu stellen. Bitte stellen Sie Fragen und fordern Sie den Dialog mit den behandelnden Ärztinnen und Ärzten ein!

Nur Patientinnen und Patienten, die ihre Grunderkrankung, deren Entstehung und den natürlichen Verlauf sowie die Chancen zu deren Verbesserung verstanden haben, können das ganze Potenzial ausschöpfen.

Konservative Therapie soll alle möglichen invasiven Maßnahmen ergänzend flankieren und bedeutet die Durchführung von Gehtraining, Einhaltung der optimalen Arzneimitteltherapie, die Kontrolle und Verbesserung des Zuckerspiegels, die Ein- stellung des Tabakkonsums, die Kontrolle und Verbesserung des Blutdrucks sowie die Optimierung von Ernährung und Körpergewicht. Mit all diesen Maßnahmen können Sie Ihr Leben verlängern, einen Herzinfarkt oder Schlaganfall verhindern, Ihre Beine erhalten und die Lebensqualität erhöhen (• Abb. 3).

\section{Fazit für die Praxis}

- In allen Stadien der peripheren arteriellen Verschlusskrankheit (PAVK) sollten konservative Maßnahmen, Verordnung von Gehtraining, das Fundament vor bzw. gemeinsam mit invasiven Behandlungen darstellen.

- Eine gewissenhafte und kritische Auseinandersetzung mit der eigenen Erkrankung erhöht die Wahrscheinlichkeit, dass alle Maßnahmen gemeinsam den Verlauf verbessern.

- Unabhängig vom Vorliegen einer Symptomatik sollte nach Erstdiagnose einer PAVK stets eine Statintherapie, ggf. mit Ezetimib, eingeleitet werden.

- Zur Abschätzung des individuellen Risikos für durchblutungsbedingte Amputationen und Tod kann der GermanVasc- 
Risikoscore genutzt werden: https://score. germanvasc.de

- Zur optimalen Arzneimitteltherapie der PAVK gehören neben den Statinen und Blutdrucksenkern in symptomatischen Stadien auch gerinnungswirksame Medikamente.

- Rauchen ist der bedeutendste Risikofaktor für die Entstehung und den Verlauf der PAVK.

- Übergewicht und Fettleibigkeit sind mit Bluthochdruck und Störungen des Zuckerstoffwechsels assoziiert. Die mediterrane Diät kann die Entwicklung einer PAVK verlangsamen und den Verlauf der Erkrankung positiv beeinflussen. Das Vorliegen eines Diabetes ist der zweitwichtigste Risikofaktor für die Verschlechterung einer PAVK.

- Die regelmäßige und konsequente Einnahme der Medikamente ist wichtig für den Behandlungserfolg.

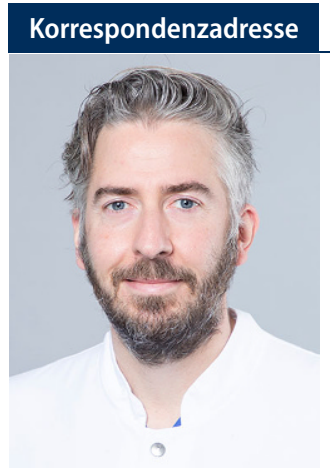

PD Dr. Christian-Alexander Behrendt

Forschungsgruppe GermanVasc, Klinik und Poliklinik für Gefäßmedizin, Universitätsklinikum Hamburg-Eppendorf

Hamburg, Deutschland

behrendt@hamburg.de

Förderung. Innovationsfond des Gemeinsamen Bundesausschuss (01VSF18035)

Funding. Open Access funding enabled and organized by Projekt DEAL.

\section{Einhaltung ethischer Richtlinien}

Interessenkonflikt. Y. Rosenberg, H. Görtz, U. Rother, C. Uhl, K. Stavroulakis, M. Pfeiffer, M. Pfeiffer-Krahl und C.-A. Behrendt geben an, dass kein Interessenkonflikt besteht.

Für diesen Beitrag wurden von den Autoren keine Studien an Menschen oder Tieren durchgeführt. Für die aufgeführten Studien gelten die jeweils dort angegebenen ethischen Richtlinien.

Open Access. Dieser Artikel wird unter der Creative Commons Namensnennung 4.0 International Lizenz veröffentlicht, welche die Nutzung, Vervielfältigung, Bearbeitung, Verbreitung und Wiedergabe in jeglichem Medium und Format erlaubt, sofern Sie den/die ursprünglichen Autor(en) und die Quelle ordnungsgemäß nennen, einen Link zur Creative Commons Lizenz beifügen und angeben, ob Änderungen vorgenommen wurden.

Die in diesem Artikel enthaltenen Bilder und sonstiges Drittmaterial unterliegen ebenfalls der genannten Creative Commons Lizenz, sofern sich aus der Abbildungslegende nichts anderes ergibt. Sofern das betreffende Material nicht unter der genannten Creative Commons Lizenz steht und die betreffende Handlung nicht nach gesetzlichen Vorschriften erlaubt ist, ist für die oben aufgeführten Weiterverwendungen des $\mathrm{Ma}$ terials die Einwilligung des jeweiligen Rechteinhabers einzuholen.

Weitere Details zur Lizenz entnehmen Sie bitte der Lizenzinformation auf http://creativecommons.org/ licenses/by/4.0/deed.de.

\section{Literatur}

1. Aboyans V, Ricco JB, Bartelink MEL et al (2018) Editor's choice-2017 ESC guidelines on the diagnosis and treatment of peripheral arterial diseases, in collaboration with the European society for vascular surgery (ESVS). Eur J Vasc Endovasc Surg 55(3):305-368. https://doi.org/10. 1016/j.ejvs.2017.07.018

2. Aboyans V, Ricco JB, Bartelink MEL et al (2018) 2017 ESC guidelines on the diagnosis and treatment of peripheral arterial diseases, in collaboration with the European society for vascular surgery (ESVS): document covering atherosclerotic disease of extracranial carotid and vertebral, mesenteric, renal, upper and lower extremityarteries. Endorsed by: the European stroke organization (ESO) the task force for the diagnosis and treatment of peripheral arterial diseases of the European society of cardiology (ESC) and of the European society for vascular surgery (ESVS). Eur Heart J39:763-816

3. Adegbola A, Behrendt C-A, Zyriax B-C et al (2021) The impact of nutrition on the development and progression of peripheral artery disease: a systematic review. Clin Nutr 41(1):49-70. https:// doi.org/10.1016/j.clnu.2021.11.005

4. Adler Al, Stevens RJ, Neil A et al (2002) UKPDS 59: hyperglycemia and other potentially modifiable risk factors for peripheral vascular disease in type 2 diabetes. Diabetes Care 25:894-899

5. American Diabetes Association (2021) 9. Pharmacologic approaches to glycemic treatment: standards of medical care in diabetes-2021. Diabetes Care 44:S111-S124

6. Anderson JW, BairdP, Davis RH Jr.etal (2009) Health benefits of dietary fiber. Nutr Rev 67:188-205

7. Anonymous (2021) ACE-Hemmer-ratiopharm $25 \mathrm{mg}$-Beipackzettel

8. Barua RS, Rigotti NA, Benowitz NL et al (2018) 2018 ACC expert consensus decision pathway on tobacco cessation treatment: a report of the American college of cardiology task force on clinical expert consensus documents. J Am Coll Cardiol 72:3332-3365

9. Bendermacher BL, Willigendael EM, Teijink JA et al (2005) Medical management of peripheral arterial disease. JThromb Haemost 3:1628-1637

10. Burns P, Gough S, Bradbury AW (2003) Management of peripheral arterial disease in primary care. BMJ 326:584-588
11. Caligiuri SP, Rodriguez-Leyva D, Aukema HM et al (2016) Dietary flaxseed reduces central aortic blood pressure without cardiac involvement but through changes in plasma oxylipins. Hypertension 68:1031-1038

12. Ciccarone E, Di Castelnuovo A, Salcuni M et al (2003) A high-score Mediterranean dietary pattern is associated with a reduced risk of peripheral arterial disease in Italian patients with type 2 diabetes. JThromb Haemost 1:1744-1752

13. Conte MS, Bradbury AW, Kolh P et al (2019) Global vascular guidelines on the management of chronic limb-threatening Ischemia. Eur J Vasc Endovasc Surg 58:S1-S109.e33

14. Cornejo Del Río V, Mostaza J, Lahoz C et al (2017) Prevalence of peripheral artery disease (PAD) and factors associated: an epidemiological analysis from the population-based screening PREdiabetes and type 2 DIAbetes (SPREDIA-2) study. PLoSONE 12:e186220

15. Cosentino F, Grant PJ, Aboyans V et al (2020) 2019ESC guidelines on diabetes, pre-diabetes, and cardiovascular diseases developed in collaboration with the EASD. Eur Heart J41:255-323

16. Cosentino F, Grant PJ, Aboyans V et al (2019) 2019ESC guidelines on diabetes, pre-diabetes, and cardiovascular diseases developed in collaboration with the EASD: the task force for diabetes, prediabetes, and cardiovascular diseases of the European society of cardiology (ESC) and the European association for the study of diabetes (EASD). Eur Heart J 41:255-323

17. Ding N, Sang Y, Chen J et al (2019) Cigarette smoking, smoking cessation, and long-term risk of 3 major atherosclerotic diseases. J Am Coll Cardiol 74:498-507

18. Dolan NC, Liu K, Criqui MH et al (2002) Peripheral artery disease, diabetes, and reduced lower extremity functioning. Diabetes Care 25:113-120

19. Duvall WL, Vorchheimer DA (2004) Multi-bed vascular disease and atherothrombosis: scope of the problem. JThromb Thrombolysis 17:51-61

20. Edel AL, Rodriguez-Leyva D, Maddaford TG et al (2015) Dietary flaxseed independently lowers circulating cholesterol and lowers it beyond the effects of cholesterol-lowering medications alone in patients with peripheral artery disease. J Nutr 145:749-757

21. Elfghi M, Jordan F, Dunne D et al (2021) The effect of lifestyle and risk factor modification on occlusive peripheral arterial disease outcomes: standard healthcare vs structured programmefor a randomised controlled trial protocol. Trials 22:138

22. Firnhaber JM, Powell CS (2019) Lower extremity peripheral artery disease: diagnosis and treatment. Am Fam Physician 99:362-369

23. Fischbach W (2019) Drug-induced gastrointestinal bleeding. Internist (Berl) 60:597-607

24. Frank U, Nikol S, Belch J et al (2019) ESVMguideline on peripheral arterial disease. Vasa 48:1-79

25. Gerhard-Herman MD, Gornik HL, Barrett $C$ et al (2017) 2016 AHA/ACC guideline on the management of patients with lower extremity peripheral artery disease: a report of the American college of cardiology/American heart association task force on clinical practice guidelines. J Am Coll Cardiol 69:e71-e126

26. Gerhard-Herman MD, Gornik HL, Barrett $C$ et al (2017) 2016 AHA/ACC guideline on the management of patients with lower extremity peripheral artery disease: a report of the American college of cardiology/American heart association task force on clinical practice guidelines. Circulation 
135(12):e726-e779. https://doi.org/10.1161/cir. 0000000000000470

27. Gupta A, Thompson D, Whitehouse A et al (2017) Adverse events associated with unblinded, but not with blinded, statin therapy in the Anglo-Scandinavian cardiac outcomes triallipid-lowering arm (ASCOT-LLA): a randomised double-blind placebo-controlled trial and its nonrandomised non-blind extension phase. Lancet 389:2473-2481

28. Harwood AE, Cayton T, Sarvanandan R et al (2016) A review of the potential local mechanisms by which exercise improves functional outcomes in intermittent claudication. Ann Vasc Surg 30:312-320

29. Jansen SC, Abaraogu UO, Lauret GJ et al (2020) Modes of exercise training for intermittent claudication. Cochrane Database Syst Rev 8:CD9638

30. Kaschwich M, Peters F, Hischke S et al (2020) Longterm incidence of cancer after index treatment for symptomatic peripheral arterial disease - a health insurance claims data analysis. Vasa 49:493-499

31. Katsouyanni K, Skalkidis Y, Petridou E et al (1991) Diet and peripheral arterial occlusive disease: the role of poly-, mono-, and saturated fatty acids. Am J Epidemiol 133:24-31

32. Kreutzburg T, Peters F, Kuchenbecker J et al (2021) Editor's choice - the GermanVasc score: a pragmatic risk score predicts five year amputation free survival in patients with peripheral arterial occlusive disease. Eur J Vasc Endovasc Surg 61:248-256

33. Kreutzburg T, Peters F, Riess HC et al (2020) Editor's choice-comorbidity patterns among patients with peripheral arterial occlusive disease in Germany: a trend analysis of health insurance claims data. Eur JVasc Endovasc Surg 59:59-66

34. Lawall H, Huppert P, Rümenapf G (2015) S3Leitlinie. Periphere arterielle Verschlusskrankheit (PAVK), Diagnostik, Therapie und Nachsorge (Registernummer 065-003)

35. Leng GC, Horrobin DF, Fowkes FG et al (1994) Plasma essential fatty acids, cigarette smoking, and dietary antioxidants in peripheral arterial disease. A population-based case-control study. Arterioscler Thromb 14:471-478

36. Mach F, Baigent C, Catapano AL et al (2020) 2019 ESC/EAS guidelines for the management of dyslipidaemias: lipid modification to reduce cardiovascular risk. Eur Heart J 41:111-188

37. Mattioli AV, Coppi F, Migaldi M et al (2017) Relationship between Mediterranean diet and asymptomatic peripheral arterial disease in a population of pre-menopausal women. Nutr Metab Cardiovasc Dis 27:985-990

38. McDermott MM (2018) Exercise rehabilitation for peripheral artery disease: a REVIEW. J Cardiopulm Rehabil Prev 38:63-69

39. Peters F, Kreutzburg T, Rieß HC et al (2020) Editor's choice-optimal pharmacological treatment of symptomatic peripheral arterial occlusive disease and evidence of female patient disadvantage: an analysis of health insurance claims data. Eur JVasc Endovasc Surg 60:421-429

40. Peters F, Kuchenbecker J, Kreutzburg T et al (2020) Long-term effectiveness and safety of initiating statin therapy after index revascularization in patients with peripheral arterial occlusive disease. J Am Heart Assoc 9:e18338

41. Piepho RW (2000) Overview of the angiotensinconverting-enzyme inhibitors. Am J Health Syst Pharm 57(1):S3-7

42. Piepoli MF, Hoes AW, Agewall S et al (2016) 2016 European guidelines on cardiovascular disease

\section{Recommendations on conservative therapy and secondary prevention of peripheral arterial disease (PAD): an evidence-based information brochure for sufferers}

Peripheral arterial disease (PAD) is considered a common disease affecting more than 230 million people worldwide and has a bad prognosis. Owing to the systemic, progressive nature of atherosclerosis and the involvement of several vascular beds, not only is there a risk of vascular amputations but also a reduction in the general life expectancy. The thorough exhaustion of conservative therapy is considered an important pillar of complementary care, but this is not always successfully implemented. Besides structured exercise training, smoking cessation, optimisation of nutrition and body weight, antihypertensive therapy, as well as antidiabetic therapy and lipid-lowering therapy, the optimal pharmacological treatment is considered a central topic. This article is aimed at providing evidence-based recommendations for the best medical treatment for PAD in layman's terms.

\section{Keywords}

Peripheral arterial disease · Prevention · Atherosclerosis · Intermittent claudication . Pharmacological treatment

prevention in clinical practice: the sixth joint task force of the European society of cardiology and other societies on cardiovascular disease prevention in clinical practice (constituted by representatives of 10 societies and by invited experts)developed with the special contribution of the European association for cardiovascular prevention \& rehabilitation (EACPR). Eur Heart J 37:2315-2381

43. Pistoia F, Cipollone F, Ferri C et al (2010) Cyclooxygenase and atherosclerosis: a smoking area.Curr Pharm Des 16:2567-2571

44. Rodrigues E, Silva I (2020) Supervised exercise therapy in intermittent claudication: a systematic review of clinical impact and limitations. Int Angiol 39:60-75

45. Rodriguez-Leyva $D$, Rodriguez-Portelles $A$, Weighell W et al (2019) The effects of dietary flaxseed on cardiac arrhythmias and claudication in patients with peripheral arterial disease. Can J Physiol Pharmacol 97:557-561

46. Ruiz-Canela M, Estruch R, Corella D et al (2014) Association of Mediterranean diet with periphera artery disease: the PREDIMED randomized trial. JAMA 311:415-417

47. Saratzis A, Jaspers NEM, Gwilym B et al (2019) Observational study of the medical management of patients with peripheral artery disease. Br J Surg 106:1168-1177

48. Seed SA, Harwood AE, Sinclair J et al (2021) A systematic review of exercise prescription in patients with intermittent claudication: does pain matter? Ann Vasc Surg 28:28

49. Song P, Rudan D, Zhu Y et al (2019) Global, regional, and national prevalence and risk factors for peripheral artery disease in 2015: an updated systematic review and analysis. Lancet Glob Health 7:e1020-e1030

50. Treat-Jacobson D, Mcdermott MM, Bronas UG et al (2019) Optimal exercise programs for patients with peripheral artery disease: a scientific statement from the American heart association. Circulation 139:e10-e33

51. Unger T, Borghi C, Charchar F et al (2020) 2020 international society of hypertension global hypertension practice guidelines. Hypertension 75:1334-1357
52. Williams B, Mancia G, Spiering W et al (2018) 2018 ESC/ESH guidelines for the management of arterial hypertension. Eur Heart J 39:3021-3104

53. Wood FA, Howard JP, Finegold JA et al (2020) N-of-1 trial of a statin, placebo, or no treatment to assess side effects. N Engl J Med 383:2182-2184

54. Zomer E, Gurusamy K, Leach R et al (2016) Interventions that cause weight loss and the impact on cardiovascular risk factors: a systematic review and meta-analysis. Obes Rev 17:1001-1011 